\title{
A Simplified Approach to the Density Functional Theory of Molecules
}

\author{
Christian Kollmar \\ Organisch-Chemisches Institut, Winterthurerstr. 190, Universität Zürich, CH-8057 Zürich
}

Z. Naturforsch. 54 a, 101-109 (1999); received December 2, 1998

\begin{abstract}
A simplified molecular orbital (MO) formalism based on density functional theory is developed. Starting from the same energy expression as Kohn-Sham theory the electronic density is expanded in terms of atomic mono-center densities. Application of the variational principle leads to a secular matrix with a particularly transparent structure which allows the definition of a resonance integral taking into account the effect of interference in a natural way. The construction of the secular matrix scales formally as $N^{2}$ instead of $N^{3}$ in the Kohn-Sham formalism with $\mathrm{N}$ being the dimension of the atomic orbital (AO) basis set.
\end{abstract}

Key words: Density Functional Theory; Molecular Orbital; Kohn-Sham Theory; Ruedenberg Approximation; Resonance Integral.

\section{Introduction}

In recent times density functional theory (DFT) enjoys an increasing popularity in the field of computational chemistry. It is based on the theorem of Hohenberg and Kohn [1] who have shown that the ground state energy of a molecule is a unique functional of the electron density $\rho$. A break-through was the idea of Kohn and Sham to obtain the electronic density from an auxiliary system of non-interacting particles [2]. An advantage of DFT as compared to Hartree-Fock theory is the inclusion of electron correlation. Moreover, the method scales formally as $N^{3}$ in contrast to the ab initio Hartree-Fock method scaling as $N^{4}$ with $N$ being the dimension of the atomic orbital (AO) basis set. Thus, larger systems become computationally tractable. The accuracy of this approach rests on the choice of appropriate exchangecorrelation energy functionals the search for which is an active field of current research [3 - 7].

In a previous contribution [8] a simplified MO formalism scaling as $N^{2}$ has been presented within the framework of Hartree-Fock theory. Using an AO basis set $\left\{\phi_{\mu_{A}}\right\}$ with the subscript $A$ denoting the atom to which the corresponding $\mathrm{AO}$ is assigned and a truncated Ruedenberg expansion [9] for the representation of diatomic overlap densities $\phi_{\mu_{A}}^{*} \phi_{\nu_{B}}$, the essence of this formalism is the following: once the

Reprint requests to Dr. Ch. Kollmar;

E-mail: kollmar@oci.unizh.ch. matrix elements $h_{\mu_{A} \mu_{A}^{\prime}}$ of the single-particle Hamiltonian between AO's located on the same atom, i.e., the diagonal blocks of the Hamiltonian matrix, have been evaluated, the matrix elements between AO's located on different atoms, i. e., the off-diagonal blocks are as [8]:

$$
\begin{aligned}
h_{\mu_{A} \nu_{B}}= & \beta_{\mu_{A} \nu_{B}} \\
& +\frac{1}{2}\left(\sum_{\mu_{A}^{\prime}} S_{\mu_{A}^{\prime} \nu_{B}} h_{\mu_{A} \mu_{A}^{\prime}} \sum_{\nu_{B}^{\prime}} S_{\mu_{A} \nu_{B}^{\prime}} h_{\nu_{B}^{\prime} \nu_{B}}\right) .
\end{aligned}
$$

$\beta_{\mu_{A} \nu_{B}}$ is the resonance integral defined previously [8] and $S_{\mu_{A} \nu_{B}}$ represents the overlap integral between AO's $\phi_{\mu_{A}}$ and $\phi_{\nu_{B}}$. The introduction of a resonance integral is of conceptual value because it takes into account the effect of interference essential for chemical bonding [10 - 12].

It is the aim of this contribution to show that the concepts mentioned above and used previously in the development of an approximate Hartree-Fock MO method [8] can be more consistently implemented in the framework of DFT for two reasons. First, the electron density is obtained from an explicit wave function in Hartree Fock theory. The Ruedenberg approximation is imposed on this density afterwards. In DFT, on the other hand, an explicit wave function never appears. All that is needed is an expression for the electronic energy as a functional of the density and a variational ansatz for the latter. Only these ingredients will be used in the following. Second, due to the non- 
locality of the exchange potential in Hartree-Fock theory, the exchange part of the Fock matrix is difficult to handle and does not fit into the scheme (1) [8]. If we can retrieve the scheme of (1) for the construction of the secular matrix in the context of DFT, the formal scaling of the method would be reduced from $N^{3}$ to $N^{2}$. This would put the present approach on the same level of computational efficiency as current semiempirical MO methods, e. g. MNDO [13].

The formal scaling of the different methods mentioned above is not directly related to the actual computational cost as a function of system size. It has been shown that integral screening substantially reduces the effective scaling of the ab initio Hartree-Fock method from its formal value of $N^{4}[14,15]$. In DFT, expansion of the density in an auxiliary basis leads to formal $N^{3}$ instead of $N^{4}$ scaling $[16,17]$. The computational efficiency of DFT can be enhanced further by using confined basis orbitals, i. e., orbitals which are strictly zero beyond a certain radius [18]. The nonselfconsistent Harris approximation [19] and related schemes [20] represent another possibility to facilitate the application of DFT to very large systems. The most important progress in this direction, however, has been achieved by generalization of the fast multipole method to Gaussian charge distributions leading to linear scaling for very large systems [21 - 24]. Note that methods based on "traditional" evaluation of two-electron integrals show only quadratic scaling for large systems in the most favorable case. However, it is not only the power of the scaling but also the prefactor that matters. In this respect, the formalism presented in this paper with the number of two-electron integrals being proportional to $N^{2}$ instead of $N^{4}$ or $N^{3}$ at the outset has an inherent computational advantage. Of course, such an approach can also benefit from linear scaling as has been demonstrated for the semiempirical AM1 method [25].

It should be noted that the theoretical basis of current semiempirical MO methods remains unclear. This gave rise to considerable work trying to clarify this problem [11, 26-29]. Especially the zero differential overlap (ZDO) approximation seems rather drastic and no clear-cut justification has been given so far [30]. Since this problem is avoided in the present approach we think that it provides a sounder basis for a semiempirical MO method than currently used schemes. We emphasize this aspect because the practical value as an ab initio method in analogy to Kohn-Sham theory remains yet to be proven and no final judgement can be given before implementation. On the other hand, semiempirical methods are more flexible because they are based on the philosophy that methodological shortcomings can be partially compensated by an appropriate parametrization. There have been efforts to deduce semiempirical MO schemes from DFT [31]. The present formalism may serve as a basis for a genuine semiempirical DFT method. Implementation on an ab initio basis, on the other hand, would also facilitate the application of DFT to very large systems.

\section{The Formalism}

Since the formalism to be developed in the following is closely related to Kohn-Sham theory we would like to review briefly the basic steps of the latter. The total electronic energy is given by the expression

$$
\begin{aligned}
E= & T_{\mathrm{S}}[\rho(\boldsymbol{r})]+\int V(\boldsymbol{r}) \rho(\boldsymbol{r}) \mathrm{d}^{3} \boldsymbol{r} \\
& +\frac{1}{2} \int \rho(\boldsymbol{r}) \rho\left(\boldsymbol{r}^{\prime}\right) \frac{1}{\left|\boldsymbol{r}-\boldsymbol{r}^{\prime}\right|} \mathrm{d}^{3} \boldsymbol{r} \mathrm{d}^{3} \boldsymbol{r}^{\prime}+E_{\mathrm{xc}}[\rho(\boldsymbol{r})] .
\end{aligned}
$$

$\rho(\boldsymbol{r})$ is the electronic density, $V(\boldsymbol{r})$ the external Coulomb potential, and $E_{\mathrm{xc}}$ the exchange-correlation energy. In Kohn-Sham theory the density is obtained from a wave function of non-interacting electrons which is simply the product of the eigenfunctions $\psi_{i}$ of a single-particle Hamiltonian. The electronic density for a closed-shell system is then given as

$$
\rho(\boldsymbol{r})=\sum_{i \text { (occ. MO's) }}\left|\psi_{i}(\boldsymbol{r})\right|^{2} .
$$

The sum on the right hand side of (3) extends over all occupied MO's and this will be the convention for all sums over $\mathrm{MO}$ indices appearing in the following. The functional $T_{\mathrm{S}}$ represents the kinetic energy of the independent-particle system which must not be identified with the kinetic energy of the real system of interacting particles. We have

$$
T_{\mathrm{S}}=2 \sum_{i} \int \psi_{i}^{*}(\boldsymbol{r})\left(-\frac{1}{2} \Delta\right) \psi_{i}(\boldsymbol{r}) \mathrm{d}^{3} \boldsymbol{r} .
$$

Applying the variational principle to (2), we obtain

$$
\begin{aligned}
\delta E= & \delta T_{\mathrm{S}}[\rho(\boldsymbol{r})] \\
& +\int\left(V(\boldsymbol{r})+V_{\text {Hartree }}(\boldsymbol{r})+V_{\mathrm{xc}}(\boldsymbol{r})\right) \delta \rho(\boldsymbol{r}) \mathrm{d}^{3} \boldsymbol{r}
\end{aligned}
$$


with

$$
V_{\mathrm{xc}}(\boldsymbol{r})=\frac{\delta E_{\mathrm{xc}}[\rho(\boldsymbol{r})]}{\delta \rho(\boldsymbol{r})}
$$

and

$$
V_{\text {Hartree }}(\boldsymbol{r})=\int \rho\left(\boldsymbol{r}^{\prime}\right) \frac{1}{\left|\boldsymbol{r}-\boldsymbol{r}^{\prime}\right|} \mathrm{d}^{3} \boldsymbol{r}^{\prime}
$$

Finally, the variational principle leads to the following eigenvalue equation

$$
\hat{h}^{\mathrm{KS}} \psi_{i}(\boldsymbol{r})=\varepsilon_{i} \psi_{i}(\boldsymbol{r})
$$

where we have introduced the Kohn-Sham singleparticle Hamiltonian

$$
\hat{h}^{\mathrm{KS}}=-\frac{1}{2} \Delta+V(\boldsymbol{r})+V_{\text {Hartree }}(\boldsymbol{r})+V_{\mathrm{xc}}(\boldsymbol{r}) .
$$

$\varepsilon_{i}$ are the MO energies. Now we make an LCAO (linear combination of atomic orbitals) ansatz for the MO's:

$$
\psi_{i}=\sum_{A} \sum_{\mu_{A}} \phi_{\mu_{A}} C_{\mu_{A} i}
$$

Using (10) in the expression for the electronic density (3) we obtain

$$
\rho(\boldsymbol{r})=\sum_{A} \sum_{\mu_{A}} \sum_{B} \sum_{\nu_{B}} P_{\nu_{B} \mu_{A}} \phi_{\mu_{A}}^{*}(\boldsymbol{r}) \phi_{\nu_{B}}(\boldsymbol{r})
$$

with the elements of the bond order matrix $\mathbf{P}$ being defined as

$$
P_{\nu_{B} \mu_{A}}=2 \sum_{i} C_{\nu_{B} i} C_{\mu_{A} i}^{*}
$$

The operator equation (8) is now transformed into a matrix equation in the $\mathrm{AO}$ basis

$$
\mathbf{h}^{\mathrm{KS}} \mathbf{C}=\mathbf{S C} \boldsymbol{\varepsilon}
$$

$\mathbf{S}$ is the overlap matrix. $\mathbf{h}^{\mathrm{KS}}$ is the matrix of the KohnSham operator (9) in the AO basis:

$$
h_{\mu_{A} \nu_{B}}^{\mathrm{KS}}=\left\langle\phi_{\mu_{A}}\left|\hat{h}^{\mathrm{KS}}\right| \phi_{\nu_{B}}\right\rangle .
$$

C is the matrix of the eigenvector coefficients appearing in (10) and $\varepsilon$ a diagonal matrix containing the $\mathrm{MO}$ energies $\varepsilon_{i}$.
In a previous paper [8] we have used a truncated Ruedenberg expansion [9] for the representation of the diatomic overlap densities appearing in (11):

$$
\begin{aligned}
\phi_{\mu_{A}}^{*} \phi_{\nu_{B}}=\frac{1}{2}( & \sum_{\mu_{A}^{\prime}} S_{\mu_{A}^{\prime} \nu_{B}} \phi_{\mu_{A}}^{*} \phi_{\mu_{A}^{\prime}} \\
& \left.+\sum_{\nu_{B}^{\prime}} S_{\mu_{A} \nu_{B}^{\prime}} \phi_{\nu_{B}^{\prime}}^{*} \phi_{\nu_{B}}\right) .
\end{aligned}
$$

Inserting (15) into (11) we obtain

$$
\begin{aligned}
\rho(\boldsymbol{r})=\frac{1}{2} & \left(\sum_{A} \sum_{\mu_{A}} \sum_{\mu_{A}^{\prime}}(S P)_{\mu_{A}^{\prime} \mu_{A}} \phi_{\mu_{A}}^{*}(\boldsymbol{r}) \phi_{\mu_{A}^{\prime}}(\boldsymbol{r})\right. \\
& \left.+\sum_{B} \sum_{\nu_{B}} \sum_{\nu_{B}^{\prime}}(P S)_{\nu_{B} \nu_{B}^{\prime}} \phi_{\nu_{B}^{\prime}}^{*}(\boldsymbol{r}) \phi_{\nu_{B}}(\boldsymbol{r})\right) .
\end{aligned}
$$

Introducing the Chirgwin-Coulson bond order matrix [32]

$$
\mathbf{N}=\frac{1}{2}(\mathbf{P S}+\mathbf{S P})
$$

(16) may be rewritten as

$$
\rho(\boldsymbol{r})=\sum_{A} \sum_{\mu_{A}} \sum_{\mu_{A}^{\prime}} N_{\mu_{A}^{\prime} \mu_{A}} \phi_{\mu_{A}}^{*}(\boldsymbol{r}) \phi_{\mu_{A}^{\prime}}(\boldsymbol{r}) .
$$

Within the context of Hartree-Fock theory, (18) clearly is an approximation to the electron density which can be justified to some extent if this density appears in a Coulomb integral. With regard to density functional theory, however, one might adopt a different point of view: here we interpret the matrix elements of $\mathbf{N}$ as variational parameters for the optimization of the ground state electron density such that the total energy reaches a minimum. We assume that the atomic basis set has been chosen flexible enough to allow the electron density to be represented with sufficient accuracy by (18). Equation (17) is retained so that the matrix elements of $\mathbf{N}$ are varied via a variation of the original density matrix $\mathbf{P}$, i.e., the MO coefficients $C_{\mu_{A} i}$ appearing in (12). Note that the electron density (18) is invariant with respect to rotations of the coordinate axes and hybridization of the $\mathrm{AO}$ basis set [33]. It is also necessary that spatial integration of the density (18) gives the total number of electrons. If we assume the AO's on the same atom 
to form an orthonormal set, this may be easily verified by integration of (18) resulting in the trace of the matrix $\mathbf{N}$ which is equal to the number of electrons.

The principal advantage of Kohn-Sham theory as compared to earlier density functional approaches is the introduction of a functional $T_{\mathrm{S}}$ (see (4)) which represents a more appropriate approximation to the kinetic energy than, e.g., the Thomas-Fermi functional for the kinetic energy. We therefore retain (4) for the functional $T_{\mathrm{S}}$ assuming that the MO's obtained from our approach ressemble the Kohn-Sham MO's. This remains to be proven in a practical implementation of the method but if the two sets of MO's are not perfectly identical, they can be expected to have at least the same nodal properties. It should be noted that (4) is not an exact representation of the kinetic energy of the interacting system, neither in Kohn-Sham theory nor in our approach. A part of the kinetic energy of the interacting system is always contained in the exchange-correlation energy functional $E_{\mathrm{xc}}$. It remains to be seen if corrections to the latter will be necessary. In the present approach, the variation of the total energy is still given by (5). The only change in comparison to Kohn-Sham theory is the ansatz (18) instead of (11) for the electron density.

From (18) and using the LCAO ansatz (10) in (4), we obtain for the variation of the density and of the kinetic energy

$$
\begin{gathered}
\delta \rho(\boldsymbol{r})=\sum_{A} \sum_{\mu_{A}} \sum_{\mu_{A}^{\prime}} \delta N_{\mu_{A}^{\prime} \mu_{A}} \phi_{\mu_{A}}^{*}(\boldsymbol{r}) \phi_{\mu_{A}^{\prime}}(\boldsymbol{r}), \\
\delta T_{\mathrm{S}}=\sum_{A} \sum_{\mu_{A}} \sum_{B} \sum_{\nu_{B}} \delta P_{\nu_{B} \mu_{A}}\left\langle\phi_{\mu_{A}}\left|-\frac{1}{2} \Delta\right| \phi_{\nu_{B}}\right\rangle .
\end{gathered}
$$

Note the formal analogy of the kinetic energy variation (20) to Kohn-Sham theory. Inserting (19) and (20) in (5), we obtain

$$
\begin{gathered}
\delta E=\sum_{A} \sum_{\mu_{A}} \sum_{B} \sum_{\nu_{B}} \delta P_{\nu_{B} \mu_{A}}\left\langle\phi_{\mu_{A}}\left|-\frac{1}{2} \Delta\right| \phi_{\nu_{B}}\right\rangle(21) \\
+\sum_{A} \sum_{\mu_{A}} \sum_{\mu_{A}^{\prime}} \delta N_{\mu_{A}^{\prime} \mu_{A}}\left\langle\phi_{\mu_{A}}\left|\hat{V}+\hat{V}_{\text {Hartree }}+\hat{V}_{\mathrm{xc}}\right| \phi_{\mu_{A}^{\prime}}\right\rangle .
\end{gathered}
$$

Using (9), we rewrite (21):

$$
\begin{aligned}
\delta E= & \sum_{A} \sum_{\mu_{A}} \sum_{B} \sum_{\nu_{B}} \delta P_{\nu_{B} \mu_{A}}\left\langle\phi_{\mu_{A}}\left|-\frac{1}{2} \Delta\right| \phi_{\nu_{B}}\right\rangle(22) \\
& +\sum_{A} \sum_{\mu_{A}} \sum_{\mu_{A}^{\prime}} \delta N_{\mu_{A}^{\prime} \mu_{A}}\left\langle\phi_{\mu_{A}}\left|\hat{h}^{\mathrm{KS}}+\frac{1}{2} \Delta\right| \phi_{\mu_{A}^{\prime}}\right\rangle .
\end{aligned}
$$

From (17) it can be seen that the variation of the elements of the Chirgwin-Coulson bond order matrix is given as

$\delta N_{\mu_{A}^{\prime} \mu_{A}}=\sum_{B} \sum_{\nu_{B}}\left(\delta P_{\mu_{A}^{\prime} \nu_{B}} S_{\nu_{B} \mu_{A}}+S_{\mu_{A}^{\prime} \nu_{B}} \delta P_{\nu_{B} \mu_{A}}\right)$.

Inserting (23) in (22) and renaming some of the indices, we obtain after some straightforward manipulations

$$
\delta E=\sum_{A} \sum_{\mu_{A}} \sum_{B} \sum_{\nu_{B}} \delta P_{\nu_{B} \mu_{A}} \tilde{h}_{\mu_{A} \nu_{B}}
$$

with

$$
\begin{aligned}
\tilde{h}_{\mu_{A} \nu_{B}}= & \left\langle\phi_{\mu_{A}}\left|-\frac{1}{2} \Delta\right| \phi_{\nu_{B}}\right\rangle \\
+ & \frac{1}{2}\left(\sum_{\mu_{A}^{\prime}}\left\langle\phi_{\mu_{A}}\left|\hat{h}^{\mathrm{KS}}+\frac{1}{2} \Delta\right| \phi_{\mu_{A}^{\prime}}\right\rangle S_{\mu_{A}^{\prime} \nu_{B}}\right. \\
& \left.+\sum_{\nu_{B}^{\prime}} S_{\mu_{A} \nu_{B}^{\prime}}\left\langle\phi_{\nu_{B}^{\prime}}\left|\hat{h}^{\mathrm{KS}}+\frac{1}{2} \Delta\right| \phi_{\nu_{B}}\right\rangle\right) .
\end{aligned}
$$

Adding the orthonormality constraints multiplied by Lagrangian multipliers to the energy variation (24), the variational condition results again in an eigenvalue equation:

$$
\tilde{\mathbf{h}} \mathbf{C}=\mathbf{S C} \boldsymbol{\varepsilon} .
$$

The reader might wonder what has been gained by solving (26) instead of (13). The advantage of this formulation becomes obvious if we take a closer look on the elements of the matrix $\tilde{\mathbf{h}}$. Let us first consider the diagonal blocks $(A=B)$. The symbol $S$ in (25) may then be replaced by a Kronecker $\delta$ because AO's on the same center are assumed to form an orthonormal set. We then obtain immediately

$$
\tilde{h}_{\mu_{A} \nu_{A}}=\left\langle\phi_{\mu_{A}}\left|\hat{h}^{\mathrm{KS}}\right| \phi_{\nu_{A}}\right\rangle \text {. }
$$

Now we consider the elements of the off-diagonal blocks $(A \neq B)$. These can be written as

$$
\begin{aligned}
\tilde{h}_{\mu_{A} \nu_{B}} & =\beta_{\mu_{A} \nu_{B}} \\
+ & \frac{1}{2}\left(\sum_{\mu_{A}^{\prime}} \tilde{h}_{\mu_{A} \mu_{A}^{\prime}} S_{\mu_{A}^{\prime} \nu_{B}}+\sum_{\nu_{B}^{\prime}} S_{\mu_{A} \nu_{B}^{\prime}} \tilde{h}_{\nu_{B}^{\prime} \nu_{B}^{\prime}}\right)
\end{aligned}
$$


with the following definition of the resonance integral $\beta$ :

$$
\begin{aligned}
\beta_{\mu_{A} \nu_{B}}= & \left\langle\phi_{\mu_{A}}\left|-\frac{1}{2} \Delta\right| \phi_{\nu_{B}}\right\rangle \\
- & \frac{1}{2}\left(\sum_{\mu_{A}^{\prime}}\left\langle\phi_{\mu_{A}}\left|-\frac{1}{2} \Delta\right| \phi_{\mu_{A}^{\prime}}\right\rangle S_{\mu_{A}^{\prime} \nu_{B}}\right. \\
& \left.+\sum_{\nu_{B}^{\prime}} S_{\mu_{A} \nu_{B}^{\prime}}\left\langle\phi_{\nu_{B}^{\prime}}\left|-\frac{1}{2} \Delta\right| \phi_{\nu_{B}}\right\rangle\right) .
\end{aligned}
$$

Looking at (28), we observe that we have now retrieved the scheme of (1) directly from the variational principle. The important point is that the off-diagonal blocks can be constructed easily once the elements of the diagonal blocks have been calculated. Only the resonance integrals need to be evaluated directly in the AO basis which is a simple thing to do. The remainder of these matrix elements is completely determined by the elements of the diagonal blocks and the overlap integrals.

The particular ansatz (18) simplifies also the calculation of the elements of the diagonal blocks which are given by the matrix elements of the Kohn-Sham Hamiltonian (9). It is important to note that only twocenter integrals appear in the Hartree term. Inserting (18) into (7) and forming the matrix elements necessary for the construction of the diagonal blocks, we obtain

$$
\begin{gathered}
\left\langle\phi_{\mu_{A}}\left|\hat{V}_{\text {Hartree }}\right| \phi_{\mu_{A}^{\prime}}\right\rangle= \\
\sum_{B} \sum_{\nu_{B}} \sum_{\nu_{B}^{\prime}} N_{\nu_{B}^{\prime} \nu_{B}}\left(\phi_{\nu_{B}} \phi_{\nu_{B}^{\prime}} \mid \phi_{\mu_{A}} \phi_{\mu_{A}^{\prime}}\right),
\end{gathered}
$$

where we have introduced the abbreviation

$$
\begin{aligned}
& \left(\phi_{\nu_{B}} \phi_{\nu_{B}^{\prime}} \mid \phi_{\mu_{A}} \phi_{\mu_{A}^{\prime}}\right)= \\
& \int \phi_{\nu_{B}}^{*}\left(\boldsymbol{r}^{\prime}\right) \phi_{\nu_{B}^{\prime}}\left(\boldsymbol{r}^{\prime}\right) \frac{1}{\left|\boldsymbol{r}-\boldsymbol{r}^{\prime}\right|} \phi_{\mu_{A}}^{*}(\boldsymbol{r}) \phi_{\mu_{A}^{\prime}}(\boldsymbol{r}) \mathrm{d}^{3} \boldsymbol{r} \mathrm{d}^{3} \boldsymbol{r}^{\prime} .
\end{aligned}
$$

Now it is obvious that the construction of the matrix $\tilde{\mathbf{h}}$ scales as $N^{2}$. The number of matrix elements of the diagonal blocks is proportional to $N$, and the number of two-electron integrals appearing in each of these matrix elements is also proportional to $N$, as can be seen from (30). The number of matrix elements of the off-diagonal blocks grows of course as $N^{2}$ but these are obtained directly from (28), i. e., they do not depend on the size of the system.

The number of matrix elements $\left\langle\phi_{\mu_{A}}\left|\hat{V}_{\mathrm{xc}}\right| \phi_{\nu_{B}}\right\rangle$ of the exchange correlation potential is also reduced in the present approach and grows only linearly with $N$ because the particular form (18) of the density eliminates the need to evaluate these matrix elements for AO's located on different centers $(A \neq B)$.

\section{Correlation Energy}

The formalism described in the previous section might also lead to a simplified approximation to, at least, the intraatomic correlation energy which is very often the dominant part [34]. This is due to the fact that the expression (18) makes it very easy to decompose the molecular electron density into contributions from individual atoms. We have

$$
\rho(\boldsymbol{r})=\sum_{A} \rho_{A}(\boldsymbol{r})
$$

with

$$
\rho_{A}(\boldsymbol{r})=\sum_{\mu_{A}} \sum_{\mu_{A}^{\prime}} N_{\mu_{A}^{\prime} \mu_{A}} \phi_{\mu_{A}}^{*}(\boldsymbol{r}) \phi_{\mu_{A}^{\prime}}(\boldsymbol{r}) .
$$

The diagonal elements $N_{\mu_{A} \mu_{A}}$ of the matrix $\mathbf{N}$ give the occupation number of the AO $\phi_{\mu_{A}}$. Empirical expressions for intraatomic correlation energies as a function of $\mathrm{AO}$ occupation numbers have been used in the literature $[34,35]$. Thus, at least in a semiempirical implementation of the present DFT scheme, one might consider the representation of the correlation energy as a function of the matrix elements $N_{\mu_{A}^{\prime} \mu_{A}}$ instead of a functional of a continuous density $\rho(\boldsymbol{r})$. This corresponds to the discretization of a continuous function. The discrete values represent the density in atomic regions in our case. Let us assume that we have an expression for the exchange-correlation energy as a function of the elements of the matrix $\mathbf{N}$ :

$$
E_{\mathrm{xc}}=f\left(N_{\mu_{A}^{\prime} \mu_{A}}\right) .
$$

It is easy to include this term into the variation of the electronic energy. It turns out that we have just to replace the matrix elements $\left\langle\phi_{\mu_{A}^{\prime}}\left|V_{\mathrm{xc}}\right| \phi_{\mu_{A}}\right\rangle$ appearing in the diagonal blocks of the matrix $\tilde{\mathbf{h}}$ in (27) by the derivatives $\partial f / \partial N_{\mu_{A}^{\prime} \mu_{A}}$. Given (34), these are much easier to evaluate than the former matrix elements which require numerical integration. 


\section{The Energy Gradient}

Since geometric optimization of molecular structures is one of the fundamental objectives in modern theoretical chemistry it is important to have analytic expressions for the derivatives of the electronic energy. Such expressions have been developed in great detail by Komornicki and Fitzgerald [36] for KohnSham DFT and can be adapted to the present formalism.

In contrast to Kohn-Sham theory, the electronic density is not given by (3) in our case. However, we may accept (3) as a formal notation for the density if it is understood that the density is not obtained by simply plugging in the Kohn-Sham MO's in (3), which leads to (11) but rather by subsequent application of the Ruedenberg approximation (15). In that case (3) is completely equivalent to (18) and we can adopt all formulae of [36] as long as they refer to the MO basis. We just have to take care that the Ruedenberg approximation is applied to all terms except the kinetic energy when making the transition from the MO to the $\mathrm{AO}$ basis.

Let us consider the gradient for a variationally optimized wavefunction as an example. Collecting the MO coefficients in a matrix $\mathbf{C}$ and following the notation of [36] we may write

$$
\mathbf{C}=\mathbf{C}^{0} \mathbf{U T} \text {. }
$$

$\mathbf{C}^{0}$ is the MO coefficient matrix at the reference geometry, $\mathbf{U}$ allows for unitary transformations of the MO's and T ensures orthogonality of the MO's for all possible geometries. Both $\mathbf{T}$ and $\mathbf{U}$ are of course identical to the unit matrix at the reference point. Denoting the derivative with respect to a parameter $\alpha$ (a nuclear coordinate, say) by an upper index, we obtain at the reference geometry:

$$
\mathbf{C}^{\alpha}=\mathbf{C}^{0}\left(\mathbf{T}^{\alpha}+\mathbf{U}^{\alpha}\right) .
$$

The derivative $\mathbf{U}^{\alpha}$ vanishes in case of a variationally optimized wavefunction. There are many ways to define the matrix $\mathbf{T}$ but it is most obvious to choose it symmetric. Then we have [37]

$$
\mathbf{T}=\left(\mathbf{C}^{0+} \mathbf{S C}^{0}\right)^{-1 / 2} .
$$

The first derivative at the reference geometry is now given by [37]

$$
\mathbf{T}^{\alpha}=-\frac{1}{2} \mathbf{C}^{0+} \mathbf{S}^{\alpha} \mathbf{C}^{0}
$$

According to (45) of [36], the energy gradient for a variationally optimized wavefunction is given by the expression

$$
\frac{\partial E}{\partial \alpha}=2 \sum_{i(\text { occ. })}\left\langle\psi_{i}\left|-\frac{1}{2} \Delta+V\right| \psi_{i}\right\rangle^{(\alpha)}+2 \sum_{i \text { (occ. })} \sum_{j(\text { occ. })}\left(\psi_{i} \psi_{i} \mid \psi_{j} \psi_{j}\right)^{(\alpha)}+2 \sum_{i(\text { occ. })}\left\langle\psi_{i}\left|V_{\text {xc }}\right| \psi_{i}\right\rangle^{(\alpha)}+4 \sum_{i \text { (occ. })} \varepsilon_{i} T_{i i}^{\alpha} .
$$

In the last term on the right hand side of (39) we have taken into account that the matrix of the singleparticle Hamiltonian is diagonal in the canonical MO basis with the diagonal matrix elements just being the MO energies $\varepsilon_{i}$. Putting the upper index $\alpha$ in parantheses indicates that only the basis functions have to be differentiated. Inserting the LCAO ansatz (10) in the matrix elements of (39), using the definition of the bond order matrix (12), and applying the Ruedenberg approximation (15) to all terms except the kinetic energy results in the expression

$$
\begin{aligned}
\frac{\partial E}{\partial \alpha}= & \sum_{A} \sum_{B} \sum_{\mu_{A}} \sum_{\nu_{B}}\left\langle\phi_{\mu_{A}}\left|-\frac{1}{2} \Delta\right| \phi_{\nu_{B}}\right\rangle^{\alpha} P_{\nu_{B} \mu_{A}}+\sum_{A} \sum_{\mu_{A}} \sum_{\mu_{A}^{\prime}} N_{\mu_{A}^{\prime} \mu_{A}}\left(\left\langle\phi_{\mu_{A}}|V| \phi_{\mu_{A}^{\prime}}\right\rangle^{\alpha}+\left\langle\phi_{\mu_{A}}\left|V_{\mathrm{xc}}\right| \phi_{\mu_{A}^{\prime}}\right\rangle^{(\alpha)}\right) \\
& +\frac{1}{2} \sum_{A} \sum_{\mu_{A}} \sum_{\mu_{A}^{\prime}} \sum_{B} \sum_{\nu_{B}} \sum_{\nu_{B}^{\prime}} N_{\mu_{A}^{\prime} \mu_{A}} N_{\nu_{B}^{\prime} \nu_{B}}\left(\phi_{\mu_{A}} \phi_{\mu_{A}^{\prime}} \mid \phi_{\nu_{B}} \phi_{\nu_{B}^{\prime}}\right)^{\alpha} \\
& +\sum_{A} \sum_{\mu_{A}} \sum_{\mu_{A}^{\prime}} \sum_{B} \sum_{\nu_{B}} \sum_{\nu_{B}^{\prime}} N_{\mu_{A}^{\prime} \mu_{A}}^{\alpha}\left\langle\phi_{\mu_{A}}\left|V+V_{\text {Hartree }}+V_{\mathrm{xc}}\right| \phi_{\mu_{A}^{\prime}}\right\rangle+4 \sum_{i \text { (occ. })} \varepsilon_{i} T_{i i}^{\alpha} .
\end{aligned}
$$

It should be noted that the elements of the matrix $\mathbf{N}$ have to be differentiated because, according to the 
definition (17), they contain overlap integrals which depend on the basis functions. Using the derivative of (17) in the second last term on the right hand side of (40), we obtain finally

$$
\begin{aligned}
\frac{\partial E}{\partial \alpha}= & \sum_{A} \sum_{B} \sum_{\mu_{A}} \sum_{\nu_{B}}\left\langle\phi_{\mu_{A}}\left|-\frac{1}{2} \Delta\right| \phi_{\nu_{B}}\right\rangle^{\alpha} P_{\nu_{B} \mu_{A}}+\sum_{A} \sum_{\mu_{A}} \sum_{\mu_{A}^{\prime}} N_{\mu_{A}^{\prime} \mu_{A}}\left(\left\langle\phi_{\mu_{A}}|V| \phi_{\mu_{A}^{\prime}}\right\rangle^{\alpha}+\left\langle\phi_{\mu_{A}}\left|V_{\mathrm{xc}}\right| \phi_{\mu_{A}^{\prime}}\right\rangle^{(\alpha)}\right) \\
& +\frac{1}{2} \sum_{A} \sum_{\mu_{A}} \sum_{\mu_{A}^{\prime}} \sum_{B} \sum_{\nu_{B}} \sum_{\nu_{B}^{\prime}} N_{\mu_{A}^{\prime} \mu_{A}} N_{\nu_{B}^{\prime} \nu_{B}}\left(\phi_{\mu_{A}} \phi_{\mu_{A}^{\prime}} \mid \phi_{\nu_{B}} \phi_{\nu_{B}^{\prime}}\right)^{\alpha}+\frac{1}{2} \sum_{A} \sum_{\mu_{A}} \sum_{B} \sum_{\nu_{B}} S_{\nu_{B} \mu_{A}}^{\alpha} \\
& \cdot\left(\sum_{\mu_{A}^{\prime}}\left\langle\phi_{\mu_{A}}\left|V+V_{\text {Hartree }}+V_{\mathrm{xc}}\right| \phi_{\mu_{A}^{\prime}}\right\rangle P_{\mu_{A}^{\prime} \nu_{B}}+\sum_{\nu_{B}^{\prime}} P_{\mu_{A} \nu_{B}^{\prime}}\left\langle\phi_{\nu_{B}^{\prime}}\left|V+V_{\text {Hartree }}+V_{\mathrm{xc}}\right| \phi_{\nu_{B}}\right\rangle\right)+4 \sum_{i \text { (occ.) }} \varepsilon_{i} T_{i i}^{\alpha} .
\end{aligned}
$$

It should be noted that the reduced number of twoelectron integrals in comparison with the Kohn-Sham formalism reduces also the cost for the evaluation of this gradient. Higher derivatives could be calculated in a similar fashion but are very lengthy and will therefore not be given here. The present approach also avoids complications arising from the fit of the density in an auxiliary basis which is necessary to achieve the formal $N^{3}$ instead of $N^{4}$ scaling of KohnSham theory [36].

\section{Discussion and Conclusion}

It is illustrative to express the total electronic energy given by (2) in terms of matrix elements in the AO basis. First, we insert the LCAO ansatz (10) and the definition (12) of the bond order matrix in the functional $T_{\mathrm{S}}$ given by (4). Using the resulting expression, (18), (29) and (17) in (2), we obtain

$$
\begin{gathered}
E=\sum_{A \neq} \sum_{B} \sum_{\mu_{A}} \sum_{\nu_{B}} \beta_{\mu_{A} \nu_{B}} P_{\nu_{B} \mu_{A}} \\
+\sum_{A} \sum_{\mu_{A}} \sum_{\mu_{A}^{\prime}} N_{\mu_{A}^{\prime} \mu_{A}}\left\langle\phi_{\mu_{A}}\left|-\frac{1}{2} \Delta+\hat{V}\right| \phi_{\mu_{A}^{\prime}}\right\rangle \\
+\frac{1}{2} \sum_{A} \sum_{\mu_{A}} \sum_{\nu_{A}^{\prime}} \sum_{B} \sum_{\nu_{B}} \sum_{\nu_{B}^{\prime}} N_{\mu_{A}^{\prime} \mu_{A}} N_{\nu_{B}^{\prime} \nu_{B}} \\
\cdot\left(\phi_{\mu_{A}} \phi_{\mu_{A}^{\prime}} \mid \phi_{\nu_{B}} \phi_{\nu_{B}^{\prime}}\right)+E_{\mathrm{xc}}[\rho(\boldsymbol{r})] .
\end{gathered}
$$

It is clear that the total energy of a molecule (including the core-core repulsion not given in (42)) has to be lower than the energy of the separate atoms which compose the molecule. One may therefore wonder which of the terms in (42) contains the energy of the chemical bonds. The balance of the different Coulomb interactions (electron-electron repulsion, core-core repulsion, electron-core attraction) is in general hard to assess, but the chemical bond is essentially due to the interference resulting from the in-phase superposition of AO's localized on different atoms, which leads to a "flattening out" of the molecular electron density as compared to a "quasiclassical" density comprising individual atomic contributions, and thus to a lowering of the kinetic energy [10 - 12]. This effect is just accounted for by the first term on the right hand side of (42), as we would like to show in the following. Using (29) and (17), we obtain

$$
\begin{aligned}
\sum_{A \neq} \sum_{B} \sum_{\mu_{A}} \sum_{\nu_{B}} \beta_{\mu_{A} \nu_{B}} P_{\nu_{B} \mu_{A}}= \\
\sum_{A} \sum_{B} \sum_{\mu_{A}} \sum_{\nu_{B}}\left\langle\phi_{\mu_{A}}\left|-\frac{1}{2} \Delta\right| \phi_{\nu_{B}}\right\rangle P_{\nu_{B} \mu_{A}} \\
-\sum_{A} \sum_{\mu_{A}} \sum_{\mu_{A}^{\prime}}\left\langle\phi_{\mu_{A}}\left|-\frac{1}{2} \Delta\right| \phi_{\mu_{A}^{\prime}}\right\rangle N_{\mu_{A}^{\prime} \mu_{A}} .
\end{aligned}
$$

It is easily seen that the first term on the right hand side of (43) corresponds to the kinetic energy of the molecule, i.e., (4) if we neglect the kinetic energy contribution to $E_{\mathrm{xc}}$. The second term on the right hand side of (43) is obtained from the first by application of the Ruedenberg approximation corresponding to a neglect of interference effects. If each diagonal block of the matrix $\mathbf{N}$ is diagonalized by a unitary transformation of the AO's of the corresponding single atoms, this term can be interpreted as a sum of the kinetic energies of individual atoms with each hybrid AO being occupied by $N_{\mu_{A} \mu_{A}}$ electrons. Thus (43) represents the difference between the kinetic energy of the molecule and that of the separate atoms. It describes the lowering of the kinetic energy resulting from interference because the first term on the right 
hand side of (43) includes interference whereas the second term neglects it. Since (43) comprises contributions from individual atom pairs $A, B$ it would be an appropriate quantity for defining the energy of a chemical bond between atoms $A$ and $B$. This illustrates the conceptual value of the resonance integral arising quite naturally in our approach.

The variational ansatz (18) for the electron density has been motivated by the Ruedenberg approximation [9] which, however, in contrast to Hartree-Fock theory, is not used explicitly in DFT. The representation of diatomic overlap densities by the Ruedenberg or the simpler Mulliken approximation [38] has been used to rationalize the physical basis of semiempirical MO methods [11]. There have been only few attempts to implement such approximations directly in ab initio Hartree-Fock methods $[39,40]$ because their numerical accuracy is hard to assess. Since the elements of the Chirgwin-Coulson bond order matrix are introduced directly as variational parameters for the electron density instead of just being used for the approximation of a density calculated from a wave function, DFT is particularly suitable for our concept developed previously in the context of Hartree-Fock theory [8]. Moreover, only local potentials appear in the single-particle Hamiltonian of density functional theory, so that the complete Hamiltonian matrix is included into the scheme of (1), whereas the non-local exchange potential of Hartree-Fock theory has to be excluded [8]. Equation (1), which has been derived here directly from the variational principle, represents something like a general scheme for approximate MO methods where only the diagonal blocks of the Hamitonian matrix need to be recalculated independently in each iterative cycle. The advantage of this transparent structure of the secular matrix will become particularly evident in the context of a crystal orbital (CO) approach for periodic structures, which will be presented in a subsequent paper.

In the implementation of the present approach one can follow two routes. First. one could implement it on an ab initio basis like the Kohn-Sham formalism. This should not encounter principal difficulties. One should just remember that all AO's on the same atom must be orthogonalized. Second, one could also consider implementation on a semiempirical level in a minimal basis as already mentioned in the introduction. In this context it is interesting to point to a structural analogy in the Hamiltonian matrices of the present DFT scheme and the most simple semiempirical MO approach, the extended Hückel method [41]. The construction of the Hamiltonian matrix in the latter method also obeys the scheme given by (1). The elements of the diagonal blocks of the extended Hückel matrix can be written [41] as

$$
h_{\mu_{A} \mu_{A}^{\prime}}^{\mathrm{eh}}=\alpha_{\mu_{A}} \delta_{\mu_{A} \mu_{A}^{\prime}} \text {. }
$$

The off-diagonal elements

$$
h_{\mu_{A} \nu_{B}}^{\mathrm{eh}}=\frac{1}{2} k S_{\mu_{A} \nu_{B}}\left(\alpha_{\mu_{A}}+\alpha_{\nu_{B}}\right)
$$

are obtained from (1) if we assume the following resonance integral:

$$
\beta_{\mu_{A} \nu_{B}}^{\text {eh }}=\frac{1}{2}(k-1) S_{\mu_{A} \nu_{B}}\left(\alpha_{\mu_{A}}+\alpha_{\nu_{B}}\right) .
$$

Thus, interference is taken into account by choosing $k>1$ in the extended Hückel method. In practice, a value of $k=1.75$ is used.

From a practical point of view, the most striking advantage of the present formalism is given by a reduction of the computational cost for the construction of the Hamiltonian matrix, which might make it worthwhile to pursue this approach. We have seen that it scales formally as $N^{2}$ instead of $N^{4}$ (ab initio Hartree-Fock) or $N^{3}$ (Kohn-Sham theory). While the computational efficiency of this approach is thus comparable to that of existing semiempirical methods it avoids the shortcomings of the ZDO approximation applied in the latter.

\section{Acknowledgement}

I would like to thank Michael C. Böhm who alerted me to expressions for intraatomic correlation energies as a function of $\mathrm{AO}$ occupation numbers. 
[1] P. Hohenberg and W. Kohn, Phys. Rev. 136, B864 (1964).

[2] W. Kohn and L. J. Sham, Phys. Rev. 140, A1133 (1965).

[3] S. J. Vosko, L. Wilk, and M. Nusair, Can. J. Phys. 58, 1200 (1980).

[4] A. D. Becke, Phys. Rev. A 38, 3098 (1988).

[5] C. Lee, W. Yang, and R.G. Parr, Phys. Rev. B 37, 785 (1988).

[6] T. Grabo and E. K. U. Gross, Chem. Phys. Lett. 240, 141 (1995).

[7] M. Filatov and W. Thiel, Int. J. Quant. Chem. 62, 603 (1997).

[8] C. Kollmar, Chem. Phys. Lett. 269, 215 (1997).

[9] K. Ruedenberg, J. Chem. Phys. 19, 1433 (1951).

[10] W. Kutzelnigg, Angew. Chem. (Intern. Ed.) 12, 546 (1973).

[11] F. Driessler and W. Kutzelnigg, Theor. Chim. Acta 43, 1,307 (1976).

[12] K. Ruedenberg, Rev. Mod. Phys. 34, 326 (1962).

[13] M. J. S. Dewar and W. Thiel, J. Amer. Chem. Soc. 99, 4899 (1977).

[14] M. Häser and R. Ahlrichs, J. Comp. Chem. 10, 104 (1989).

[15] D. L. Strout and G. E. Scuseria, J. Chem . Phys. 102, 8448 (1995).

[16] E. J. Baerends, D. E. Ellis, and P. Roos, Chem. Phys. 2, 41 (1973).

[17] B. I. Dunlap, J. W. D. Connolly, and J. R. Sabin, J. Chem. Phys. 71, 3396 (1979).

[18] O. F. Sankey and D. J. Niklewski, Phys. Rev. B40, 3979 (1989).

[19] J. Harris, Phys. Rev. B 31, 1770 (1985).

[20] G. Seifert, D. Porezag, and Th. Frauenheim, Int. J. Quant. Chem. 58, 185 (1996).

[21] C. A. White, B. G. Johnson, P. M. W. Gill, and M. Head-Gordon, Chem. Phys. Lett. 253, 268 (1996).
[22] M. C. Strain, G. E. Scuseria, and M. J. Frisch, Science 271, 51 (1996).

[23] D. Sánchez-Portal, P. Ordejon, E. Artacho, and J. M. Soler, Int. J. Quant. Chem. 65, 453 (1997).

[24] M. Challacombe and E. Schwegler, J. Chem. Phys. 106, 5526 (1997).

[25] A. D. Daniels, J. M. Millam, and G. E. Scuseria, J. Chem. Phys. 107, 425 (1997).

[26] P.G. Nelson, J. Chem. Res. (M), 1701 (1980).

[27] S. de Bruijn, Int. J. Quant. Chem. 25, 367 (1984).

[28] K. Jug, Theor. Chim. Acta 14, 91 (1969).

[29] W. Thiel, Adv. Chem. Phys. 93, 703 (1996).

[30] C. Kollmar and M. C. Böhm, Theor. Chim. Acta 92, 13 (1995).

[31] E. Lindholm and S. Lundqvist, Physica Scripta, 32, 220 (1985).

[32] B. H. Chirgwin and C. A. Coulson, Proc. Roy. Soc. London A201, 196 (1950).

[33] P. J. A. Ruttink, Theor. Chim. Acta 6, 83 (1966).

[34] A. M. Olés, F. Pfirsch, and M. C. Böhm, Chem. Phys. 120, 65 (1988).

[35] (a) A. M. Olés, F. Pfirsch, W. Borrmann, P. Fulde, and M. C. Böhm, Chem. Phys. 106, 27 (1986);

(b) A. M. Olés, F. Pfirsch, P. Fulde, and M. C. Böhm, J. Chem. Phys. 85, 5183 (1986).

[36] A. Komornicki, G. Fitzgerald, J. Chem. Phys. 98, 1398 (1993).

[37] H. F. King, R. N. Camp, and J. W. McIver (Jr.), J. Chem. Phys. 80, 1171 (1984).

[38] R. S. Mulliken, J. Chim. Phys. 46, 497,675 (1949).

[39] R. D. Brown, F. R. Burden, and G. R. Williams, Theor. Chim. Acta 18, 98 (1970).

[40] W. Koch, Z. Naturforsch. 48 a, 819 (1993).

[41] R. Hoffmann, J. Chem. Phys. 39, 1397 (1963). 